Participating countries were the UK, Germany, Switzerland, Australia and New Zealand. Infants were randomised to receive either hormonal therapy and vigabatrin or hormonal therapy alone. A second stage randomization allowed hormonal treatment to be allocated as either prednisolone or tetracosactide depot. Minimum doses were: vigabatrin $100 \mathrm{mg} / \mathrm{kg} / \mathrm{day}$, prednisolone $40 \mathrm{mg}$ per day, or IM tetracosactide depot $0.5 \mathrm{mg}$ on alternate days. Hormonal treatment was continued for 2 weeks and then weaned over 2 weeks. Vigabatrin was continued for 3 months and then weaned over a month. The early primary outcome measure was cessation of spasms on and between days 14 and 42. Analysis is by intention to treat. 377 children were enrolled and early clinical outcome data will be available on 376 (1 case withdrew). 186 were allocated hormonal therapy and vigabatrin and 191 were allocated hormonal therapy alone. We will report on the primary clinical outcome and serious adverse clinical events. Developmental outcome at 18 months of age will be reported in a subsequent paper. To date this is by far the largest treatment study of infantile spasms ever undertaken.

\section{G60 STARTING ANTIEPLIPELTIC MEDICATIONS BY NON SPECIALISTS: WHAT ARE THE HAZARDS?}

NT El Tantawi, D Salama, B Hassanein, K Fathy. Neurolohy, Mansoura University Children Hospital, Mansoura, Egypt

\subsection{6/archdischild-2015-308599.59}

Background Initial prescription of antiepileptic drugs (AEDs) for newly diagnosed epileptic children should be done by a specialist. However many new patients start their treatment by paediatrician witout expertice in epilepsy.

Aim To study the prescription of (AEDs) in children before they were referred to specialist (paediatric neurologist).

Methods This was a prospective study. Six hundred children referred for the first time to the epilepsy clinic in a tertiary university hospital were recruited. Detailed (AEDs) history was retrieved from the parents in their first visit regarding the number of seizure after which they start treatment, AEDs prescribed at the beginning of diagnosis and in the following 12 months, if the drug has changed and the reasons. Patients were classified as truly epileptic and non epileptic after being reviewed by 2 neurologists.

Results Truly epileptic patients represented $65 \%$ of the newly referred patients. Of those, $45 \%$ have started one or more of AEDs before referral. Thirty nine percent started after their first seizure. Monotherapy was initiated in $65 \%$ of epileptic patients. Soduim Valproate $(65.1 \%)$ was the most frequently prescribed AED followed by Levetiracetam $(41.0 \%)$ and topiramate (38.0\%). The combination between Soduim valproate and Levetiracetam as a starting therapy was the most common. Twenty five percent of patients have changed the initial (AEDs) in the first 3 months of starting treatment. Worsening of seizures and non availability of the medication were the most common causes of changing (AEDs).

The non epileptic patients included diagnosis of: febrile seizures, breath holding attacks, pallid attacks and self stimulating. When offered to withdraw treatment after explanation of the condition by two neurologists, 28\% refused to stop AEDs.

Conclusion Starting AEDs by non specialist paediatrician has the hazards of wrong diagnosis, inappropriate starting and changing of AEDs.

\section{G61 A FIVE-YEAR RETROSPECTIVE REVIEW OF THE MANAGEMENT OF CHILDHOOD ENCEPHALITIS}

${ }^{1} \mathrm{MA}$ Iro, ${ }^{2} \mathrm{E}$ Hulbert-Powell, ${ }^{3} \mathrm{~S}$ Ling. ' Oxford Vaccine Group, Department of Paediatrics, University of Oxford and the National Institute for Health Research Oxford Biomedical Research Centre, Oxford, UK; ${ }^{2}$ Department of Paediatrics, Poole Hospitals NHS Foundation Trust, Poole, UK; ${ }^{3}$ Department of Paediatrics, Basingstoke and North Hampshire Hospitals NHS Trust, Basingstoke, UK

\subsection{6/archdischild-2015-308599.60}

Background Early diagnosis and institution of appropriate treatment are key to improving outcomes from encephalitis. This study aimed to review the management of children with encephalitis in South East England.

Methods A retrospective review of clinical notes and electronic patient records (EPR) was conducted in between April 2013 and January 2014 across four hospitals (3 district general and 1 tertiary). Children aged $0-17$ years who were admitted between 2008 and 2012 and had a discharge diagnosis of encephalitis were identified through the clinical coding department. Data on clinical features, investigation and treatment were collected.

Findings Medical records of thirty-four children were reviewed. A lumbar puncture was performed in 31 (91\%) cases. A complete CSF order set (defined as CSF: white cell count, red blood cell count, gram stain, paired CSF and serum glucose and protein level) was requested in 21/30 (70\%) cases. A complete PCR panel (CSF sent for the 3 main viral causes of encephalitis: enterovirus, herpes simplex and varicella zoster virus) was performed in 20/30 (67\%) cases. The median time to performing a brain CT scan was $24 \mathrm{~h}$ (range 23-168) and $48 \mathrm{~h}$ (range 24240) for brain MRI scan. The first dose of intravenous aciclovir was administered within $48 \mathrm{~h}$ for thirty-three (97\%) cases. The prescribed aciclovir dose was incorrect in fifteen (44\%) cases. The median duration of aciclovir treatment for children with enteroviral (EV) encephalitis was 5 days (IQR 2.5-5). The median length of hospital stay for the EV encephalitis group was 6 days (IQR 5.8-7.3). Six children with EV encephalitis received aciclovir treatment beyond $48 \mathrm{~h}$ due to non-availability of PCR test result. Children with EV encephalitis had a further median stay of 1.5 days (IQR 1.0-3.8) after availability of PCR result. Conclusion The management of childhood encephalitis is heterogeneous. The recently published UK guidelines may help standardise practice. Widespread availability of PCR testing across hospitals and improved turnaround time could lead to early diagnosis and substantial cost saving from reduced hospital stay for infants with enteroviral encephalitis. Urgent steps are needed to reduce intravenous aciclovir prescribing errors.

\section{G62(P) A CRITICAL APPRAISAL OF THE LITERATURE ON THE BENEFITS OF GASTROSTOMY FEEDING, COMPARED TO ORAL FEEDING, IN CHILDREN WITH CEREBRAL PALSY}

P Mehta, C Acerini. Department of Paediatrics, Addenbrooke's Hospital, Cambridge University Hospitals NHS Foundation Trust, Cambridge, UK

\subsection{6/archdischild-2015-308599.61}

Aims To assess whether gastrostomy is a beneficial intervention for feeding children with cerebral palsy, compared with only oral feeding, for:

1. promoting growth and weight gain in children who have dysphagia and inadequate nutrition; and 